UCRL-JC-131411

PREPRINT

\title{
Observation of Photoexcited Emission Clusters in the Bulk of KDP and Laser Conditioning under 355-nm Irradiation
}

\author{
S. G. Demos \\ M. Staggs \\ M. Yan \\ H. B. Radousky \\ J. J. De Yoreo \\ This paper was prepared for submittal to the \\ Society of Photo-Optical Instrumentation Engineers Conference Proceedings \\ Boulder, $\mathrm{CO}$ \\ September 28-October 1, 1998
}

December 15, 1998

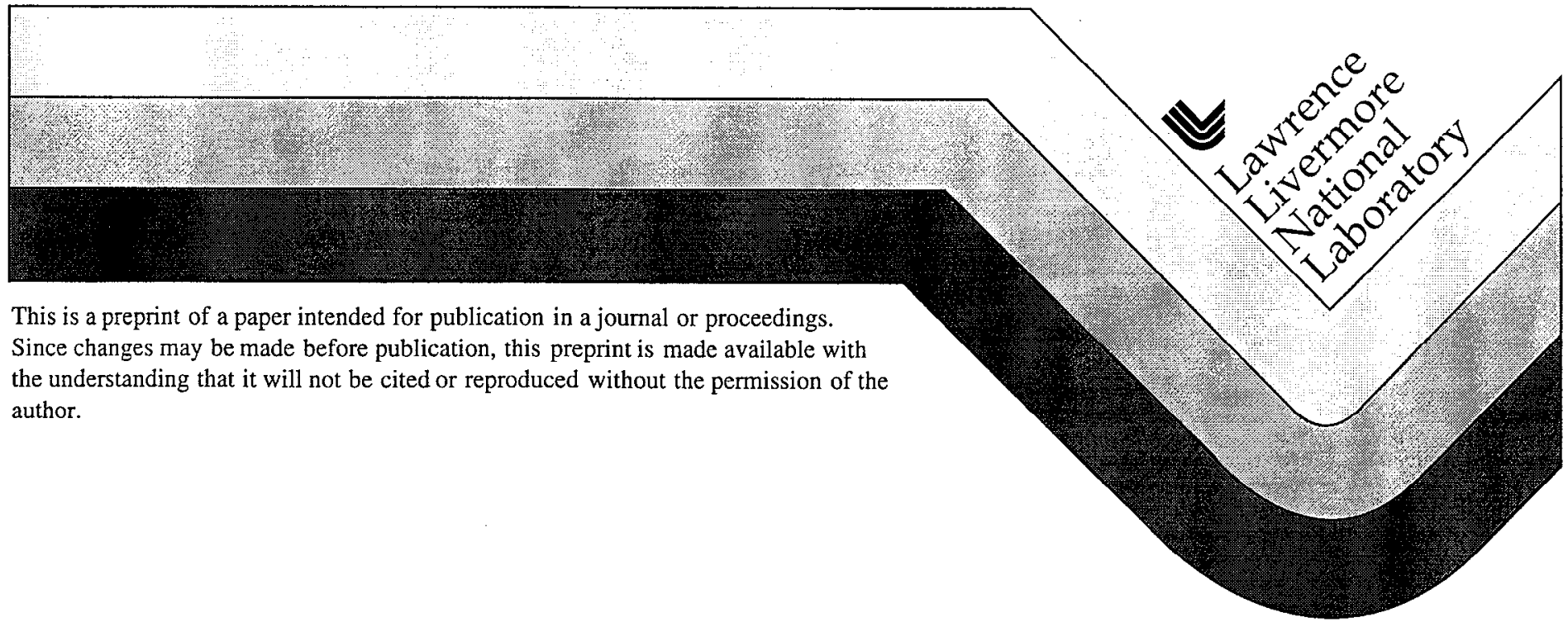




\section{DISCLAIMER}

This document was prepared as an account of work sponsored by an agency of the United States Government. Neither the United States Government nor the University of California nor any of their employees, makes any warranty, express or implied, or assumes any legal liability or responsibility for the accuracy, completeness, or usefulness of any information, apparatus, product, or process

disclosed, or represents that its use would not infringe privately owned rights. Reference herein to any specific commercial product, process, or service by trade name, trademark, manufacturer, or otherwise, does not necessarily constitute or imply its endorsement, recommendation, or favoring by the United States Government or the University of California. The views and opinions of authors expressed herein do not necessarily state or reflect those of the United States Government or the University of California, and shall not be used for advertising or product endorsement purposes. 


\title{
Observation of Photoexcited Emission clusters in the bulk of KDP and Laser Conditioning under 355-nm Irradiation
}

\author{
S. G. Demos, M. Staggs, M. Yan, H. B. Radousky and J. J. De Yoreo \\ Lawrence Livermore National Laboratory, PO Box 808, Livermore, CA 94580. \\ Tel.: (925) 423 3388, Fax: (925) 4232463
}

\section{ABSTRACT}

Defect clusters in the bulk of large KDP crystals are revealed using a microscopic fluorescence imaging system and $\mathrm{CW}$ laser illumination. Exposure of the crystal to high power $355-\mathrm{nm}, 3-\mathrm{ns}$ laser irradiation leads to a significant reduction of the number of observed optically active centers. The initially observed defect cluster concentration is approximately $10^{4}-10^{6}$ per $\mathrm{mm}^{3}$ depending on the crystal growth method and sector of the crystal. The number of defect clusters can be reduced by a factor of $10^{2}$ or more under exposure to $355-\mathrm{nm}$ laser irradiation while their average intensities also decreases. Spectroscopic measurements provide information on the electronic structure of the defects.

Key words: KDP, Fluorescence Microscopy, Spectroscopy, damage

\section{INTRODUCTION}

The formation of defects in dielectric materials during growth and subsequently to electromagnetic or particle irradiation have attracted a great deal of attention in the scientific community due to their importance in processing and performance of materials. In many dielectric crystals, the presence of electronic defects in their lattice becomes the origin of optical features of technological importance such as laser crystals. ${ }^{1}$ In other cases, the presence of defects is associated with reduced performance of the material. It is currently believed by many researchers that bulk damage in non-absorbing optical materials is initiated by the generation of free carriers which, in turn, absorb more light and lead to irreversible cascade processes. ${ }^{2-5}$ Laser induced damage in KDP crystals at relatively low irradiation levels is accompanied with the formation of micron-size pits suggesting that there are initiation sites in the bulk of the crystal responsible for localized enhanced absorption under laser photoexcitaton leading to damage. This indicates that the presence of uniformly distributed atomic defects or impurities in the bulk may not be directly associated with laser induced damage. In order to explain the bulk damage characteristics one needs to assume the presence of clusters of impurities or defects that can undertake the role of the damage initiation sites. For this reason, the development of a technique that can by used to detect and image optically active centers located in the bulk of transparent materials may be of great help for better understanding the laser induced damage mechanism.

In this work, photoexcited defect clusters in the bulk of KDP crystals are detected using a microscopic fluorescence imaging systern with 1 micron spatial resolution. The dynamics of these optically active defect clusters under high-power $355-\mathrm{nm}$ laser irradiation is revealed. The experimental results show that the number of defect clusters is reduced by a factor of $10^{2}-10^{3}$ under exposure to $355-\mathrm{nm}$ laser irradiation. Spectroscopic measurements provide information regarding the spectral characteristics of the photons that give rise to the recorded images and the electronic structure of the observed defect clusters. Damage testing experiments for the correlation of the observed defect clusters to laser induced damage were also curried out.

\section{EXPERIMENTAL SET-UP}

The experimental setup is shown in fig. 1. The third harmonic of a 3-ns pulsewidth, Q-switched, Nd:YAG laser is overlapped with the $\mathrm{CW}$ output of an argon laser operating at 514-nm or 488- $\mathrm{nm}$. The two overlapped and collimated beams are propagating along the z-axis (optical axis) of the KDP crystal and focused in the bulk of the crystal using a 7.5 -cm focal length cylindrical lens. The argon laser was used as the illumination source for the acquisition of the microscopic fluorescence images of the optically active centers. The high-power, $355-\mathrm{nm}$ laser was used for the laser conditioning experiments. The imaging system is positioned along the $y$-axis of the crystal and perpendicular to the direction of illumination The imaging system is composed of a long working distance microscope objective followed by a X5 magnification zoom lens The images were recorded using a liquid nitrogen cooled $C C D$ detector. The microscope objectives used in this work have a magnification power of X20 and X100 and working distances of 20-mm and 6-mm respectively. In this arrangement, $1 \mu \mathrm{m}^{2}$ of an object at the image plane is projected and recorded on 4 pixels of the $\mathrm{CCD}$. The imaging plane of the microscopic system is overlapped with the focal plane of the cylindrical lens that delivers the illumination beam into the sample. This configuration ensures that an object illuminated by the laser beam is imaged and recorded by the microscopic imaging system 


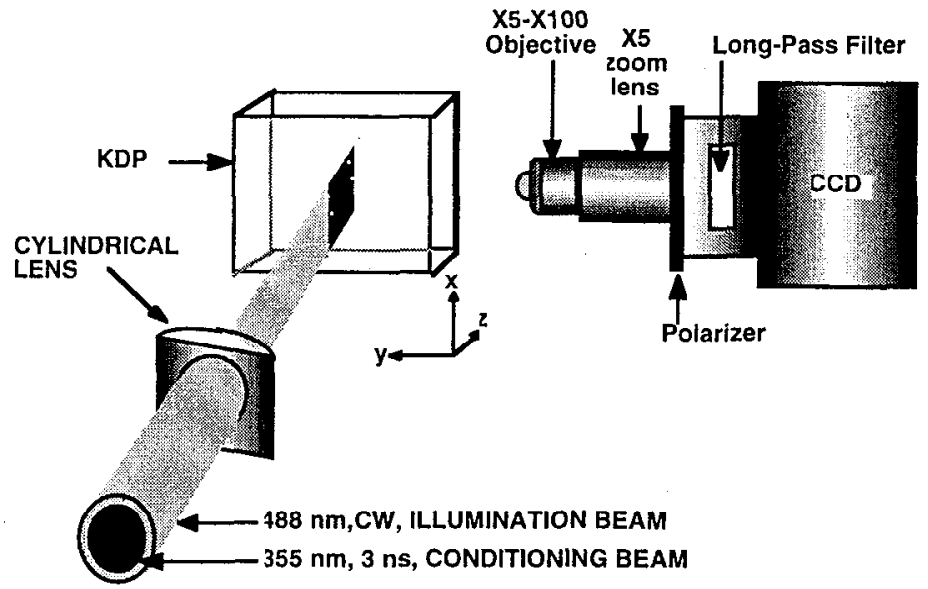

Figure 1. Schematic diagram of the microscopic fluorescence imaging experimental setup. Details are provided in the text.

The imaging depth is determined by the $\approx 25 \mu \mathrm{m}$ width of the illumination slit beam at the focal plane of the cylindrical lens. The polarization vectors of both laser beams are parallel to the $\mathrm{x}$-axis. An analyzer followed by a $640-\mathrm{nm}$ or a $700-\mathrm{nm}$ long-wavelength-pass filter are positioned between the CCD detector and the zoom lens. The analyzer with its polarization vector parallel to the $\mathrm{x}$-axis is used so that the recorded photons have polarization only perpendicular to the optical axis of the crystal and avoid image distortions due to its birefringence. The optical long-wavelength-pass filter is used in order to select imaging photons from inside the emission band of the optically active centers and discriminate against the Raman scattering signal of the bulk ${ }^{6}$ as well as the laser light scattering inside the crystal. The 700-nm long-pass (LP) filter was used under 514-nm illumination and the 640-nm LP under 488-nm illumination. The samples utilized were conventionally grown and fast grown KDP crystals. ${ }^{7,8}$ The experiments were performed at room temperature.

\section{RESULTS}

Fig. 2 shows microscopic fluorescence images using the X 20 microscope objective and 514-nm illumination. Fig. 2a shows a $200 \times 200 \times 25 \mu \mathrm{m}^{3}$ section of a fast grown KDP crystal while fig. $2 \mathrm{~b}$ shows a $200 \times 200 \times 25 \mu \mathrm{m}^{3}$ section of a conventionally grown KDP crystal. These images show the presence of emission clusters located inside the bulk of the crystal. The imaging microscopic lens in this set of measurements was kept slightly off-focus so that the image of an emission cluster appeared as a point surrounded by a characteristic ring in order to discriminate against noise registered by
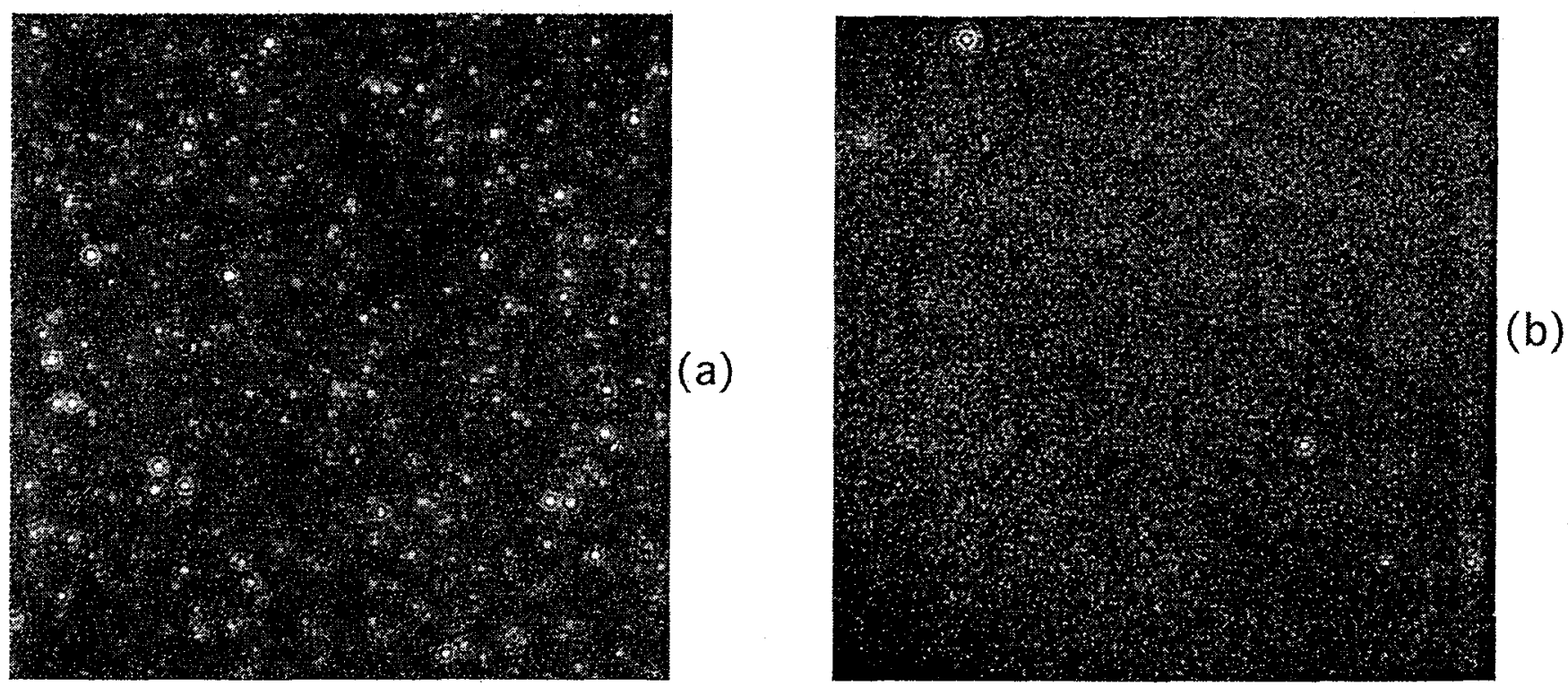

Figure 2. Microscopic fluorescence images originating from a $200 \times 200 \times 25 \mu \mathrm{m}^{3}$ section of a) a fast grown and b) a conventionally grown KDP crystal using the X20 microscope objective and 514-nm illumination.. 

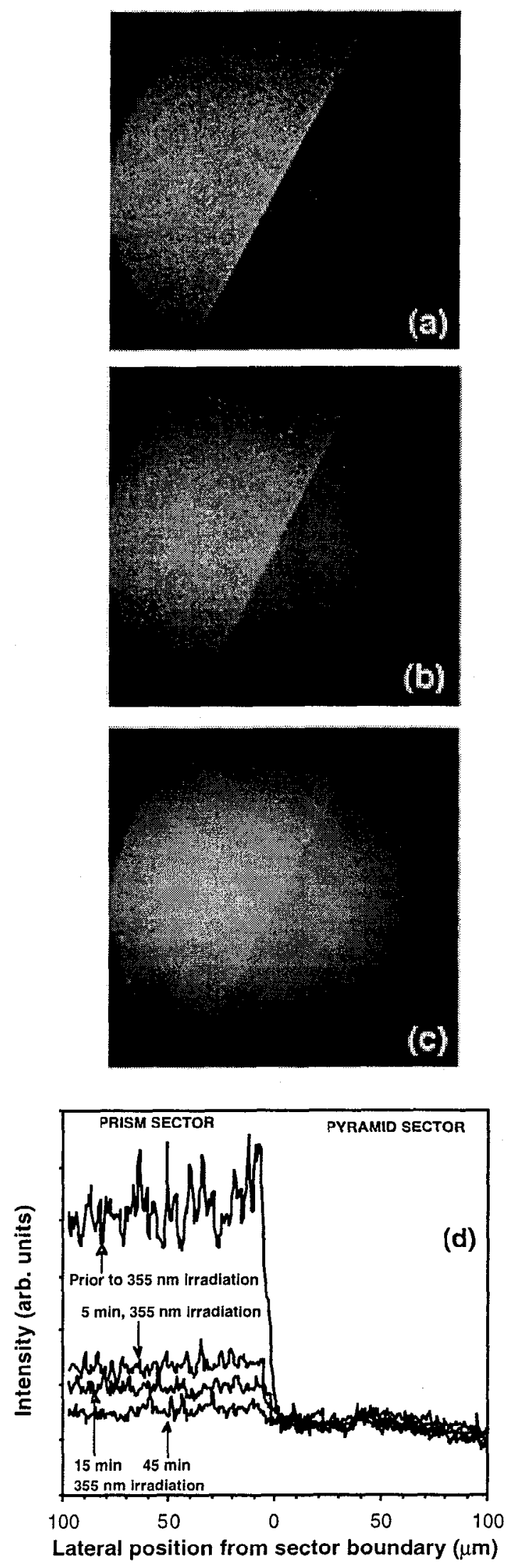

the detector. The above images were obtained using 1.5 Watts, 514$\mathrm{nm}, \mathrm{CW}$ laser illumination, a 700-nm long wavelength pass filter and a CCD exposure time of $10 \mathrm{~min}$. The features observed in fig. 2 are repeatable from site to site and from crystal to crystal. The intensities of the observed emission clusters vary with the "brightest" ones exhibiting intensities of $\approx 200$ counts while the recorded background (uniform) intensity is $\approx 60$ counts. The concentration of the observed emission clusters is very different in the images shown in figs. $2 \mathrm{a}$ and $2 \mathrm{~b}$, indicating a dependence on the crystal growth method.

Experiments were performed using fast grown KDP crystals. A strikingly large difference in the concentration of the emission clusters in the two crystals sectors ${ }^{7,8}$ was observed. The concentration of the observed emission clusters in the prismatic (material grown on $\{100\}$ faces) sector is $\approx 3 \times 10^{5}$ per $\mathrm{mm}^{3}$ while in the pyramidal (material grown on $\{101\}$ faces ) sector is $\approx 3 \times 10^{4}$ per $\mathrm{mm}^{3}$. The remarkable dissimilarity in the emission cluster concentration in the two crystal sectors allows for the observation of the sector boundary. This is demonstrated in fig. $4 \mathrm{a}$ where the fluorescence image of a $500 \mathrm{X} 500 \mathrm{X}$ $25 \mu^{3}$ section across the sector boundary of a fast grown KDP crystal is shown obtained using the $X 20$ microscope objective. The sector boundary is oriented at 45 degrees from the horizontal direction of the image plane with the prismatic sector located in the upper-left part of the image and the pyramidal sector located in the lower-right part of the image. This image was obtained prior to any UV irradiation of the sample. The sample was thereafter illuminated with $355-\mathrm{nm}$ pulses of average power of $\approx 1 \mathrm{~J} / \mathrm{cm}^{2}$ obtained from the Nd:YAG laser operating at a repetition rate of $100 \mathrm{~Hz}$. Figs. $4 \mathrm{~b}$ and $4 \mathrm{c}$ show the fluorescence images obtained following 5 minutes and 45 minutes, respectively, after irradiation of the same section of the sample with the $355-\mathrm{nm}$ laser pulses. These images show the significant reduction of the emission cluster concentration in the pyramidal sector resulting in the reduction of the intensity difference between the two crystal sectors. This conditioning effect is better depicted in fig. $4 d$ which shows the sum of the digitized intensity profile of 10 consecutive horizontal pixel-lines at the middle of images obtained following exposure to $\approx 1$ $\mathrm{J} / \mathrm{cm}^{2}, 355-\mathrm{nm}$ laser irradiation to different time intervals. These profiles demonstrate the conditioning effect which leads to an almost identical intensity level across the two crystal sectors. The conditioning effect observed in the prismatic sector occurs in the pyramidal sector as well but it is less intense due to the smaller emission cluster concentration and image intensity background. Figure $4 \mathrm{~d}$ indicates that the main portion of the conditioning takes place in the initial 5 or less minutes of irradiation followed by a slower rate of conditioning.

Figure 3. a) A microscopic fluorescence image obtained using the $\mathrm{X} 5$ microscope objective of a $1500 \times 1500 \times 25 \mu \mathrm{m}^{3}$ section of a fast grown KDP crystal at the boundary between prismatic (upper left) and pyramidal (lower right) sectors. Image of the same section following $b$ ) $5 \mathrm{~min}$. and c) $45 \mathrm{~min} 355-\mathrm{nm}$ irradiation with average fluence of $\approx 1 \mathrm{~J} / \mathrm{cm}^{2}$. d) The digitized intensity profiles across a horizontal 10 pixel stripe at the middle of the images of figs. $4 \mathrm{a}-\mathrm{c}$. 
The difference in the emission cluster concentration in the two crystal sectors allows for the extrapolation of their spectral characteristics. Using narrow band filters under $473-\mathrm{nm}$ and $458-\mathrm{nm}$ illumination, the average image intensity in the two crystal sectors was measured. The spectral profiles of the recorded intensities in the prism and pyramid sectors are shown in fig. 4. The increased intensity at lower wavelengths is due to Raman scattering from the bulk. The intensity of the spectral profiles from the two sectors of the crystal is different in the $560-\mathrm{nm}$ to $800-\mathrm{nm}$ spectral region. The difference spectrum shown in fig. 4 reveals the spectral profile of the optical signal that gives rise to the intensity difference in the two crystal sectors. The spectral characteristics of the difference spectra obtained under 458 and $473-\mathrm{nm}$ photoexcitation are practically identical. This result indicates that the spectral differences in the two crystal sectors are due to emission by the optically active centers and not to Raman scattering which it would have caused shifting of the spectral profile of the difference spectrum to follow the change of the illumination wavelength.
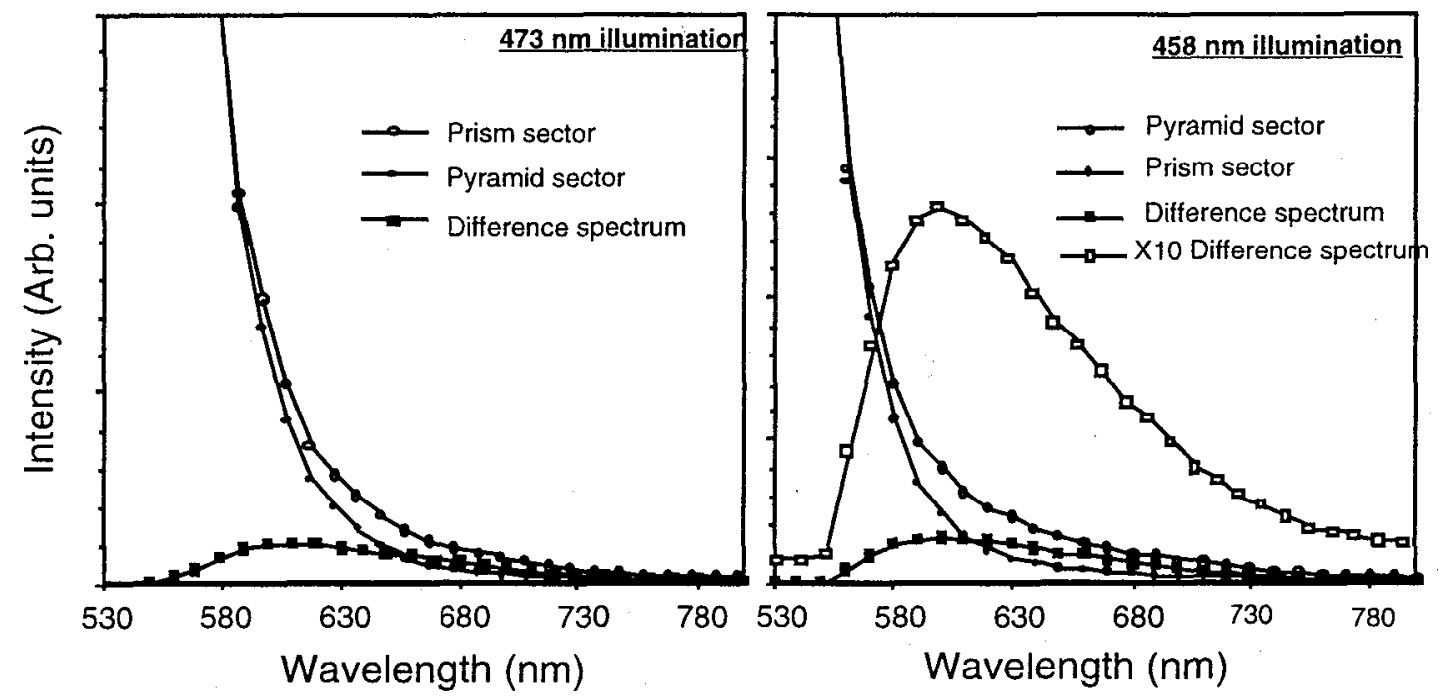

Figure 4. Spectral profiles in the 530 to $800-\mathrm{nm}$ spectral region from the prism and pyramid sectors and their difference spectrum (with the intensity enhanced by a factor of 10) under 473-nm (left) and 458-nm (right) laser excitation.

The number of emission clusters observed in the bulk of KDP using microscopic fluorescence imaging (see figs. 2 and 3) is significantly reduced when the sample is exposed to $355-\mathrm{nm}$ high-power laser irradiation. This conditioning effect is best depicted in fig. 3d. Previous work in KDP has suggested an analogous phenomenon where the damage threshold of KDP increases when the sample is irradiated with high power $355-\mathrm{nm}$ laser pulses. ${ }^{9}$ This similarity raises the question if the observed lattice defect clusters are responsible for the initiation of damage in KDP. To address this question, damage testing experiments were performed. A microscopic fluorescence image of a section of the crystal was recorded using $488-\mathrm{nm} \mathrm{CW}$
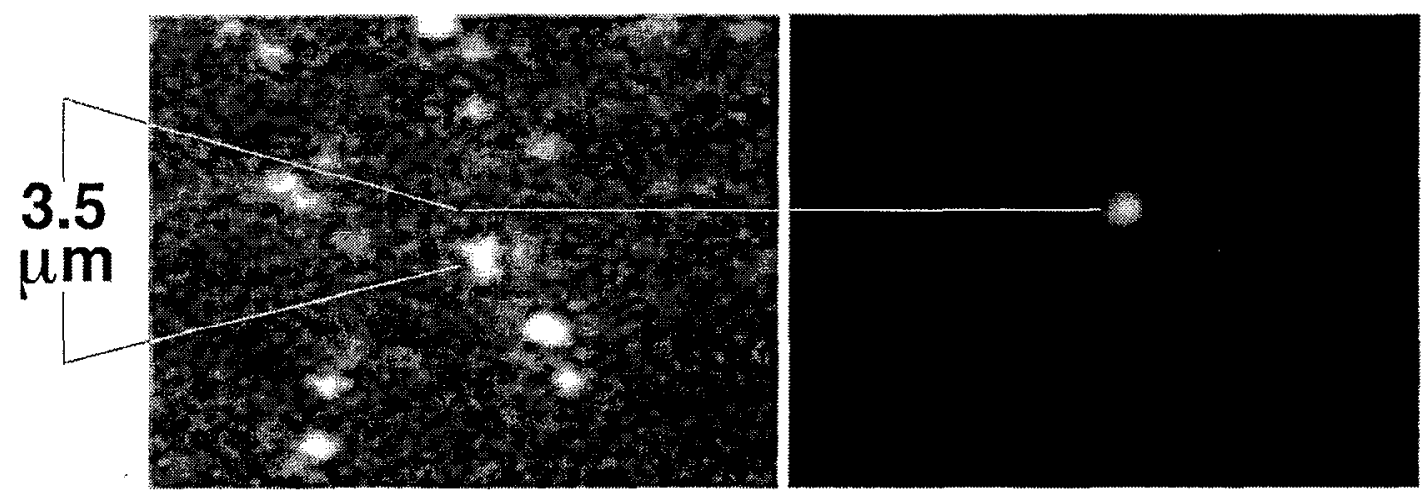

Figure 5. A section of the crystal was recorded using the microscopic fluorescence imaging system (left) and then was irradiated by a single 355-nm, 3-ns pulse. The scattering light image (right) shows the presence of a damage site. 
illumination and thereafter, the exact section of the crystal was irradiated with a single $355-\mathrm{nm}, 3 \mathrm{~ns}$ high power pulse using the experimental set-up shown in fig. 1. The Long-Pass filter was then removed from the microscopic system and a scattering-light image of the same section of the crystal was obtained to reveal the presence of laser induced damage. If damage was detected, the location of the damage site was correlated to the microscopic fluorescence image first recorded. This experimental procedure is exemplified in fig. 5 where the fluorescence image of a section of the crystal is shown on the left and the scattering-light image following a single $355-\mathrm{nm}, 3-\mathrm{ns}, \approx 8 \mathrm{~J} / \mathrm{cm}^{2}$ pulse is shown on the right. The experiment shown in figure 5 indicates that damage occurred at a location that was not occupied by an emission cluster. In general, the experimental results showed no direct correlation between the locations of the emission clusters and laser induced damage.

\section{DISCUSSION}

The experimental results shown in figs 2,3 and 4 demonstrate the ability of the technique to image optically active centers and provide important information regarding the distribution and dynamic behavior of defect clusters or particles inside a transparent optical material. The discrete emission clusters in the bulk of KDP crystals is accompanied with the presence of an emission background that is not perfectly uniform. The images shown in fig. 2 indicate that the conventionally grown KDP crystals have smaller emission cluster concentrations than the fast grown crystals. In addition, the images shown in fig. 3 indicate that within the same crystal, the emission cluster concentration in the pyramidal sector is $\approx 100$ times larger than in the prismatic sector. The overall measured particle concentration is $10^{4}-10^{6}$ per $\mathrm{mm}^{3}$ depending on the crystal growth method and crystal sector.

The large difference of the emission cluster concentration in the two crystal sectors reflects the difference in the crystal growth conditions in the two regions. It is known that the pyramidal sector has a much smaller impurity ion concentration than the prismatic sector due to the difference in the incorporation of impurities into the crystal during growth. ${ }^{10}$ The impurity ion density in the prismatic sector of fast grown KDP crystals used in this work is on the order of $\rho_{\mathrm{KDP}} \approx 10^{14} / \mathrm{mm}^{3}$. The density of the emission clusters in the fast grown KDP crystals observed in this work is $\rho_{\text {em.clu. }} \approx 10^{6} / \mathrm{mm}^{3}$. Thus, there is in average, one emission cluster for every $10^{8}$ impurity ions in the crystal. This indicates that the observed clusters are not due to phenomena associated with the presence of individual impurity ions. However, it is known that impurity ions promote the formation of defects ${ }^{1}$. This may be the reason for the large difference in the observed number of emission clusters in the two crystal sectors where the impurity ion concentration is very different. It is logical to believe that the observed emission clusters arise from foreign particles or most likely defect clusters in the bulk of the crystal. Uniformly distributed impurity ions or defects should contribute in the observed emission background.

The investigation of the reduction of the number of emission clusters under high power 355-nm irradiation indicated that this process takes place at a faster rate at the beginning exhibiting a nearly logarithmic dependence on the number the 355-nm "conditioning" pulses. Figure $4 \mathrm{~d}$ indicates that the main portion of the conditioning takes place in the initial 5 or less minutes of irradiation followed by a slower rate of conditioning. Moreover, the conditioning rate becomes larger when the energy of the $355-\mathrm{nm}$ pulses is increased. In addition to the decrease of the number of emission clusters, the intensity of the uniform background of the microscopic fluorescence images also decreases under exposure to high power $355-\mathrm{nm}$ irradiation indicating the presence of "unclustered" defect centers in the material. The average intensity of the individual centers also decreases under exposure to the $355-\mathrm{nm}$ pulsed irradiation. The conditioning effect observed in the prismatic sector occurs in the pyramidal sector as well.

Recently it was reported that intense $355-\mathrm{nm}$ or 266-nm laser irradiation of KDP crystals at room temperature leads to the formation of a broad transient optical absorption band in the $300-\mathrm{nm}$ to $650-\mathrm{nm}$ spectral region. ${ }^{11,12}$ This laser induced transient absorption was assigned to the formation of $\left(\mathrm{HPO}_{4}\right)^{-}$centers that are initiated by multi-photon absorption followed by the transport of a hydrogen atom. ${ }^{11}$ This hypothesis is supported by most recent experimental results using electronparamagnetic-resonance and resonance Raman scattering spectroscopy. ${ }^{13,14}$ The experimental technique employed in this work does not allow for a positive identification of the defects responsible for the fluorescence images obtained. However, the spectral profile of the difference spectrum shown in fig. 4 may be interpreted as the red-shifted emission arising from defect formations similar or identical to those that give rise to the 300-650-nm transient absorption spectra under high-power laser illumination suggesting the presence of $\left(\mathrm{HPO}_{4}\right)^{-}$defect clusters. It must be pointed out that the crystals studied were not exposed to any prior laser or other type of irradiation. The only possibility for prior exposure to irradiation of the samples is via naturally occurring radiation.

The emission spectrum of the optically active defect clusters observed in the bulk of KDP crystals indicates that a corresponding absorption spectrum should be present for photon energies of $2 \mathrm{eV}$ or higher. The laser conditioning effect shown in fig. 3 also indicates intense optical and electronic activity of the defects under $355-\mathrm{nm}$ irradiation. According to 
what is considered as a possible mechanism for laser-induced damage by the scientific community, these defect clusters can provide the necessary intermediate steps for multiphoton ionization and laser damage. However, the laser damage testing experiments have suggested no direct correspondence of the location of the emission clusters to damage sites. These is a number of possible reasons for this incongruity. One reason might be that light absorbing defects of different origin might also be present and are not observed by the imaging system because of low emission under photoexcitation due to stronger nonradiative relaxation. Another possible explanation might be that the transient defect distribution during the 3-ns time interval of high intensity laser irradiation might be different than the steady state distribution observed using our microscopic fluorescence imaging system under low-peak-intensity laser photoexcitation at 514-nm. The copious alteration on the location and intensity of defect clusters on images obtained prior and after a single near-damage-threshold 355-nm pulse where clusters disappear and new ones appear indicate an intense transient activity. A third reason could be that the defect clusters observed under 514-nm illuminations are not the same with the ones that are mostly active under 355-nm irradiation. Due to quantum confinement effects, the absorption spectrum may be dependent on the size of the optically active nano-particles. Utilizing the visible lines of the argon laser for imaging we may be failing to photoexcite the defect clusters that are more important which absorb light at 355 . Our future research effort is going to be coordinated to address these questions

\section{CONCLUSION}

In conclusion, the microscopic fluorescence imaging investigation of KDP crystals under CW, 514-nm laser excitation revealed the presence of optically active defect clusters located in the bulk of the crystal. The observed defect cluster concentration varies between $10^{4}-10^{6}$ per $\mathrm{mm}^{3}$ depending on the crystal growth method and sector of the crystal. Exposure of the crystal to high power, 355-nm, 3-ns, laser irradiation results in the reduction of the number of observed emission clusters. The spectroscopic measurements indicate that the absorption band of these defects is at photon energies higher than $2 \mathrm{eV}$. Laser damage testing experiments indicated no direct correlation of the observed defect clusters to damage induced by a high power $355-\mathrm{nm}$ laser pulses.

\section{ACKNOWLEDGMENTS}

This work was supported under the auspices of the U.S. Department of Energy by the Lawrence Livermore National Laboratory under contract N0. W-7405-ENG-48 through the Institute for Laser Science and Applications.

\section{REFERENCES}

1. T. T. Basiev, S. B. Mirov, "Room temperature tunable color center lasers", Laser Science and Technology: An International Handbook, V. 16, S. S. Letokhov, C. V. Shank, Y. R. Shen and H. Walther Eds., Harwood Academic publishers, 1994

2. N. Bloembergen, Laser-induced Electric Breakdown in Solids, IEE J. Quantum Electron. QE-10, 375, (1974).

3. S. C. Jones, P. Braunlich, R. T. Casper, X. A. Shen and P. Kelly, Recent, progress on laser-induced modifications and intrinsic bulk damage of wide-gap optical materials, Opt. Engineering, 28, 1039 (1989).

4. B. C. Stuart, M. D. Feit, S. Herman, A. M. Rubenchic, B. W. Shore and M. D. Perry, Nanosecond-to-femtosecond laser induced breakdown in dielectrics, Phys. Rev. B, 53, 1749 (1996).

5. R. M. OConnell, Onset Threshold Analysis of Defect-driven surface and bulk laser damage, Appl. Opt., 31, 4143.(1992).

6. C. Y. She, T. W. Broberg and David F. Edwards, Raman Spectra of $\mathrm{KH}_{2} \mathrm{PO}_{4}$, Phys. Rev. B, 4, 1580, (1971).

7. N. P. Zaitseva, J. J. De Yoreo, M. R. Dehaven, R. L. Vital, K. E. Montgomery, M. Richardson, and L. J. Atherton, Rapid growth of large-scale $(40-55 \mathrm{~cm}) \mathrm{KH}_{2} \mathrm{PO}_{4}$ crystals, J. Crystal Growth, 180, 255 (1997).

8. K. Fujioka, S. Matsuo, T. Kanabe, H. Fujita, M. Nakatsuka, Optical properties of rapidly grown KDP crystal improved by thermal conditioning. J. Crystal Growth, 181, 265 (1997).

9. J. Swain, S. Stokowski, D. Milam, F. Rainer, Improving the Bulk Laser Damage Resistance Of Potassium Dihydrogen Phosphate Crystals By Pulsed Laser Irradiation, Appl. Phys. Lett., 40, 350 (1982)

10. M. Yan, R. Torres, M. Runkel, B. Woods, I. Hutcheon, N. Zaisteva and J. J. DeYoreo, SPIE, 2966, 11, (1996).

11. J. E. Davis, R. S. Hughes Jr. and H. W. H. Lee, Investigation of optically generated transient electronic defects and protonic transport in hydrogen-bonded molecular solids - isomorphs of $\mathrm{KH}_{2} \mathrm{PO}_{4}$, Chem. Phys. Lett. 207, 540 (1993).

12. C. D. Marshall, S. A. Payne, M. A. Henesian, J. A. Speth, and H. T. Powell, ultraviolet-induced transient absorption in polassium dihydrogen phosphate and its influence on frequency conversion, J. Opt. Soc. Am. B, 11, 774 (1994).

13, S. D. Setzler, K. T. Stevens, L. E. Halliburton, M. Yan, N. P. Zaitseva, J. J. DeYoreo, Hydrogen atoms in $\mathrm{KH}_{2} \mathrm{PO}_{4}$ crystals, Phys. Rev. B, 57, 2643 (1998).

14. S. G. Demos, M. Yan, M. Staggs, J. J. DeYoreo, and H. B. Radousky, Raman scattering investigation of $\mathrm{KH}_{2} \mathrm{PO}_{4}$ subsequent to high fluence laser irradiation. Appl. Phys. Lett., 72, 2367 (1998). 